Pacific Journal of Mathematics

LIAISON OF A UNION OF SKEW LINES IN P ${ }^{4}$ 


\title{
LIAISON OF A UNION OF SKEW LINES IN $\mathbf{P}^{4}$
}

\author{
JUAN C. MigLIORE
}

\begin{abstract}
At the moment we are woefully lacking a sufficient condition for two subschemes in $\mathbf{P}^{n}$ to be in the same liaison class, except for the case of codimension 2. On the other hand, a theorem of Schenzel gives a good necessary condition in any codimension $<n$. In this article we first give a new proof of this theorem. We then apply this result to certain curves in $\mathbf{P}^{4}$ by considering hyperplane sections. Specifically, if $C$ is a collection of $t$ skew lines in $\mathrm{P}^{4}$, we ask when $C$ can be linked to another set of skew lines under the "extreme" conditions where $C$ is degenerate and where $C$ is "general".
\end{abstract}

Partly because of the absence of a sufficient condition in general, most results to date fall into one of the following two special cases: restricting to codimension 2 subschemes, often in $\mathbf{P}^{3}$ (cf. for example [B], [BM], [LR], [M1], [M2], [R1], [R2], [Su]) or restricting to arithmetically Cohen-Macaulay subschemes (cf. for example [KMU], [H], [PS], [U]). Schenzel's necessary condition ([SI]) is a generalization of a theorem of Hartshorne [R1]. The proof given in this article is similar in nature to the proof of Hartshorne's original theorem. It is worth noting that the converse to the Hartshorne-Schenzel theorem is false (cf. Remark 1.3.3). Thus one must look elsewhere for at least part of the sufficient condition(s).

Presumably the simplest situation outside of the two special cases just mentioned is that of curves in $\mathbf{P}^{4}$. After re-proving the Hartshorne-Schenzel theorem in $\S 1$, we apply it together with the techniques of [M1] to the question of when a set $C$ of $t$ skew lines in $\mathbf{P}^{4}$ can be linked to another set of skew lines. In $\S 2$ we consider the case where $C$ is degenerate, and $\S \S 3,4$ and 5 treat the case where $C$ is a "general" set of a skew lines. This generalizes a result of [M1] about unions of skew lines in $\mathbf{P}^{3}$. The idea now is that for $t \geq 4$, the Hartshorne-Rao module (suitably generalized) of $C$ provides so much information about $C$ that we can "weed out" all the sets of lines not linked to $C$. Interestingly, for $t=2$ and $t=3$ we do not have enough information to give a full answer. Again we feel the absence of a sufficient condition for liaison.

Some interesting connections with liaison of unions of lines in $\mathbf{P}^{3}$ emerge. For example if $C$ is a degenerate union of $t \geq 3$ skew lines in $\mathbf{P}^{4}$ (and in fact for more general degenerate curves) then all the degenerate curves in the liaison class of $C$ lie in the same hyperplane. Furthermore, $C$ 
is not linked to any non-degenerate union of lines. Hence one has reduced to the case of liaison in $\mathbf{P}^{3}$. If $C$ is a general collection of $t \geq 5$ skew lines, on the other hand, the results are almost identical to those in $\mathbf{P}^{3}$ in that $C$ is not linked to any other union of skew lines.

1. The machinery. In this section we give another proof of the Schenzel-Hartshorne theorem and recall the techniques of [M1] which we will use here.

Throughout this paper, $k$ shall denote an algebraically closed field. Also, $\mathbf{P}^{n}=\mathbf{P}_{k}^{n}$ and $S=k\left[X_{0}, \ldots, X_{n}\right](n \geq 3)$. (After Remark 1.3 we will take $n=4)$. If two schemes $V$ and $V^{\prime}$ are directly linked by a complete intersection $X$, we write $V \sim{ }_{X} V^{\prime}$. If they are linked we write $V \sim V^{\prime}$.

For any sheaf $\mathscr{F}, \quad H_{*}^{i}(\mathscr{F})=\oplus_{i} H^{i}\left(\mathbf{P}^{n}, \mathscr{F}(j)\right)$ and $h^{i}(\mathscr{F})=$ $\operatorname{dim}_{k} H^{i}\left(\mathbf{P}^{n}, \mathscr{F}\right)$. For any $k$-vector space $W, W^{*}=\operatorname{Hom}_{k}(W, k)$. For any graded $S$-module $M, M^{\vee}=\operatorname{Hom}_{k}(M, k)$. Finally, $\left(\mathbf{P}^{n}\right)^{*}=\{$ hyperplanes in $\left.\mathbf{P}^{n}\right\}$ is the dual projective space.

Let $V$ be a locally Cohen-Macaulay, equidimensional scheme of dimension $r$ in $\mathbf{P}^{n}$, where $1 \leq r \leq n-2$. Let $\left(M^{i}\right)(V)=H_{*}^{i}\left(\mathbf{P}^{n}, \mathscr{I}_{V}\right)$ for $1 \leq i \leq r$. $\left(M^{i}\right)(V)$ is a graded $S$-module.

We now turn to the Hartshorne-Schenzel theorem. It should be remarked that Chiarli has proved this theorem in dimension 1 using a similar approach (cf. [C]). The proof given below was motivated by conversations and correspondence with A. Geramita.

ThEOREM 1.1 (Hartshorne-Schenzel, [R1], [SI]). Let $V \sim{ }_{X} W$ by a complete intersection $X$, where $I_{X}=\left(F_{1}, \ldots, F_{n-r}\right)$ and $\operatorname{deg} F_{i}=d_{i}$. Let $d=\sum d_{i}$. Then

$$
\left(M^{r-i+1}\right)(W) \cong\left(M^{i}\right)^{\vee}(V)(n+1-d) \quad \text { for } 1 \leq i \leq r .
$$

Proof. We have a projective resolution

$$
\begin{aligned}
& 0 \rightarrow F_{n} \rightarrow F_{n-1} \rightarrow F_{n-2} \rightarrow \cdots \rightarrow \quad F_{2} \rightarrow F_{1} \rightarrow S \rightarrow S / I_{V} \rightarrow 0 \\
& \searrow \nearrow \quad \searrow \nearrow \searrow \nearrow \\
& G_{n-1} \quad G_{2} \quad I_{V} \\
& \nearrow \searrow \\
& 0 \quad 0 \\
& \begin{array}{lll}
0 & 0 & 0
\end{array}
\end{aligned}
$$

Sheafifying gives

$$
\begin{array}{cccc}
0 \rightarrow \mathscr{F}_{n} \rightarrow \mathscr{F}_{n-1} \rightarrow \mathscr{F}_{n-2} \rightarrow \cdots \rightarrow & \mathscr{F}_{2} \rightarrow \mathscr{F}_{1} \rightarrow \mathcal{O} \rightarrow \mathcal{O}_{V} \rightarrow 0 \\
\searrow \nearrow & \searrow \nearrow \searrow \nearrow \\
\mathscr{G}_{n-1} & \mathscr{G}_{2} \mathscr{I}_{V} \\
\nearrow \searrow & \nearrow \searrow \nearrow \searrow \\
0 \quad 0 & 0 & 0 \quad 0
\end{array}
$$


Then

$$
\begin{aligned}
& 0 \rightarrow \mathscr{G}_{n-r} \rightarrow \mathscr{F}_{n-r-1} \rightarrow \cdots \rightarrow \mathscr{F}_{2} \rightarrow \mathscr{F}_{1} \rightarrow \mathscr{I}_{V} \rightarrow 0 \\
& \searrow \nearrow \quad \searrow \nearrow \\
& \mathscr{G}_{n-r-1} \quad \mathscr{G}_{2}
\end{aligned}
$$

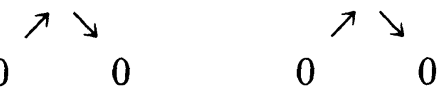

Observe that $\mathscr{G}_{n-r}$ is locally free since $V$ is locally Cohen-Macaulay. Hence Ferrand's mapping cone procedure is applicable (cf. [PS]) and we have the exact diagram

$$
\begin{aligned}
& 0 \rightarrow \mathscr{G}_{n-r} \quad \rightarrow \quad \mathscr{F}_{n-r-1} \quad \rightarrow \cdots \rightarrow \quad \mathscr{F}_{1} \quad \rightarrow \mathscr{I}_{V} \rightarrow 0 \\
& \uparrow \quad \rightarrow \quad \uparrow \quad \uparrow \\
& 0 \rightarrow \mathcal{O}(-d) \rightarrow \oplus^{n-r} \mathcal{O}\left(-a_{i}\right) \rightarrow \cdots \rightarrow \oplus^{n-r} \mathcal{O}\left(-d_{i}\right) \rightarrow \mathscr{I}_{X} \rightarrow 0
\end{aligned}
$$

where $a_{i}=d_{1}+\cdots+d_{i-1}+d_{i+1}+\cdots+d_{n-r}$. Dualizing and twisting by $-d$ and splitting off redundant terms gives

$$
\begin{aligned}
& 0 \rightarrow \mathscr{F}_{1}^{\vee}(-d) \rightarrow \oplus^{n-r} \mathscr{O}\left(d_{t}-d\right) \oplus \mathscr{F}_{2}^{\vee}(-d) \rightarrow \cdots \rightarrow \oplus^{n-r} \mathcal{O}\left(-d_{l}\right) \oplus \mathscr{G}_{n-r}^{\vee}(-d) \rightarrow \mathscr{I}_{W} \rightarrow 0 \\
& \begin{array}{ll}
\searrow \nearrow & \searrow \nearrow \\
\mathscr{K}_{2} & \mathscr{X}_{n-r-1}
\end{array} \\
& 0^{\nearrow \searrow} 0^{\nearrow \searrow} 0^{\nearrow} 0
\end{aligned}
$$

Now, by taking cohomology on the short exact sequences beginning with the penultimate one and working leftward, we have

$$
\begin{aligned}
H_{*}^{1}\left(\mathscr{K}_{n-r-1}\right) & \cong H_{*}^{2}\left(\mathscr{K}_{n-r-2}\right) \\
& \cdot \\
& \cdot \\
& \cdot \\
& =0 H_{*}^{n-r-2}\left(\mathscr{K}_{2}\right) \quad\left(\mathscr{F}_{2} \text { is free }\right) \\
& \cdot \\
& \cdot \\
& \cdot \\
H_{*}^{r+1}\left(\mathscr{K}_{n-r-1}\right) & \cong H^{r+2}\left(\mathscr{K}_{n-r-2}\right) \\
& \cdot \\
& \cdot \\
& \cong H_{*}^{n-2}\left(\mathscr{K}_{2}\right) \\
& =0 .
\end{aligned}
$$


Therefore we have

$$
\begin{array}{rlrl}
\left(M^{r-i+1}\right)(W) & =H_{*}^{r-i+1}\left(\mathscr{I}_{W}\right) & \\
& \cong H_{*}^{r-i+1}\left(\mathscr{G}_{n-r}^{\vee}(-d)\right) & & (\text { by }(1.1 .3)) \\
& \cong H_{*}^{n-r+i-1}\left(\mathscr{G}_{n-r}(d-n-1)\right)^{*} & & (\text { Serre duality }) \\
& \cong H_{*}^{n-r+i-2}\left(\mathscr{G}_{n-r-1}(d-n-1)\right)^{*} & & (\text { by }(1.1 .2)) \\
& \cdot & \\
& \cdot & \\
& \cdot & & \text { (by }(1.1 .2)) \\
& \cong H_{*}^{i+1}\left(\mathscr{G}_{2}(d-n-1)\right)^{*} & & (\text { by }(1.1 .2)) \\
& \cong H_{*}^{i}\left(\mathscr{I}_{V}(d-n-1)\right)^{*} & & \\
& =\left(M^{i}\right)^{\vee}(V)(n+1-d) & &
\end{array}
$$

(Note that these isomorphisms preserve not only a string of $k$-vector spaces but actually the module structure, by functoriality.)

Corollary 1.2 (Chiarli, [C]). Let $C$ be locally Cohen-Macaulay, equidimensional curve in $\mathbf{P}^{n}$. Let $C \sim{ }_{X} C^{\prime}$ by a complete intersection $X$, where $I_{X}=\left(F_{1}, \ldots, F_{n-1}\right)$ and $d=\sum \operatorname{deg} F_{i}$. Then

$$
M(C) \cong M\left(C^{\prime}\right)^{\vee}(n+1-d)
$$

where $M(C)=\left(M^{1}\right)(C)$.

REMARKS 1.3.

1.3.1. When $n=3$, Corollary 1.2 is Hartshorne's original theorem and $M(C)$ is called the Hartshorne-Rao module of $C$.

1.3.2. The $\left(M^{i}\right)(V)$ provide invariants of even liaison: if $V$ and $W$ are evenly linked then up to shifts we have $\left(M^{i}\right)(V) \cong\left(M^{i}\right)(W)$. If $V$ and $W$ are oddly linked then up to shifts we have $\left(M^{r-i+1}\right)(W) \cong\left(M^{i}\right)(V)^{\vee}$.

1.3.3. The converse to Theorem 1.1 is false in general, even in the form of the last remark. For example, it is known that there exist arithmetically Cohen-Macaulay curves in $\mathbf{P}^{4}$ which are not in the liaison class of a complete intersection (cf. for example [KMU]). But for any arithmetically Cohen-Macaulay curve, $M(C)=0$. So $M(C)$ does not determine the liaison class.

Now, in the case of curves in $\mathbf{P}^{4}$ we need to study the graded module $M(C)=H_{*}^{1}\left(\mathscr{I}_{C}\right)$. This involves both the dimensions of the various components and the maps between the components induced by homogeneous polynomials. 
For the former the main tool is the exact sequence

$$
0 \rightarrow \mathscr{I}_{C}(n) \rightarrow \mathcal{O}_{\mathbf{P}^{4}}(n) \rightarrow \mathcal{O}_{C}(n) \rightarrow 0
$$

which yields the long exact sequence

$$
0 \rightarrow I(C)_{n} \rightarrow S_{n} \rightarrow H^{0}\left(\mathcal{O}_{C}(n)\right) \rightarrow M(C)_{n} \rightarrow 0 .
$$

For the maps, it is enough to consider those corresponding to linear forms $L \in S_{1}$; that is, we have homomorphisms

$$
\varphi_{n}: S_{1} \rightarrow \operatorname{Hom}\left(M(C)_{n}, M(C)_{n+1}\right) .
$$

To study these we must consider two cases. If $L$ does not vanish on any component of $C$ then we use the long exact sequence associated to the exact sequence

$$
0 \rightarrow \mathscr{I}_{C}(n) \rightarrow \mathscr{I}_{C}(n+1) \rightarrow \mathscr{I}_{C \cap H}(n+1) \rightarrow 0
$$

where $H$ is the hyperplane corresponding to $L$ and the inclusion map is multiplication by $L$. If $L$ vanishes on a component of $C$ then we use either the following (simplest case) or a variant thereof: say $C=Y \cup \lambda$ where $\lambda$ is a line (reduced) which is disjoint from $Y$, and $L$ vanishes on $\lambda$ but no other component. Then

$$
\begin{aligned}
& 0 \rightarrow \mathscr{I}_{C}(n) \rightarrow \mathscr{I}_{Y}(n) \rightarrow \mathcal{O}_{\lambda}(n) \rightarrow 0 \text { and } \\
& 0 \rightarrow \mathscr{I}_{Y}(n) \rightarrow \mathscr{I}_{C}(n+1) \rightarrow \mathscr{I}_{C \cap H}(n+1) \rightarrow 0 .
\end{aligned}
$$

(The inclusion map in the second sequence is multiplication by $L$.) Note that $C \cap H$ consists of $\lambda$ together with a finite set of points in $H=\mathbf{P}^{3}$, and that the composition $\mathscr{I}_{C}(n) \rightarrow \mathscr{I}_{Y}(n) \rightarrow \mathscr{I}_{C}(n+1)$ induces the homomorphism $\varphi_{n}(L)$.

Now, while $\operatorname{dim} M(C)_{n}$ does not depend on the choice of $L$ for any $n$, the rank of $\varphi_{n}(L)$ does. Furthermore, the collection of those $L$ for which $\varphi_{n}(L)$ has a given rank is an isomorphism invariant and hence an even liaison invariant. Also, with suitable re-indexing it is also an invariant of odd liaison. These invariants can be viewed as subvarieties of the dual projective space $\left(\mathbf{P}^{4}\right)^{*}$ and there are formulas for the expected codimension and degree of these subvarieties. In many cases we can identify these invariants using only (1.1.5) and (1.1.6), without explicitly describing $\varphi_{n}$. This is our technique here. See [M1] for further details.

2. Degenerate skew lines. The goal of this section is to prove the following:

THEOREM 2.1. Let $C$ be $a$ union of $t \geq 3$ skew lines lying in $a$ hyperplane $H=\mathbf{P}^{3}$ which corresponds to a linear form $L \in S_{1}$. Let $C^{\prime}$ be any set of skew lines in $\mathbf{P}^{4}$. If $C \sim C^{\prime}$ then $C^{\prime}$ also lies in $H$. Furthermore, they are linked in $\mathbf{P}^{4}$ if and only if they are linked in $\mathbf{P}^{3}$. 
Once this is proved, we need only recall the following theorem from [M1]:

Theorem 2.2. Let $C$ be a union of $t \geq 3$ skew lines in $\mathbf{P}^{3}$ and let $C^{\prime}$ be an arbitrary union of a skew lines in $\mathbf{P}^{3}$.

(a) If $C$ lies on a quadric surface $Q$ then $C \sim C^{\prime}$ if and only if $t=s$ and $C^{\prime}$ also lies on $Q$.

(b) If $C$ does not lie on a quadric surface then $C$ is not evenly linked to any other set of skew lines, and is oddly linked to at most one other.

(C) A general set of $t \geq 4$ skew lines in $\mathbf{P}^{3}$ is not linked to any other set of skew lines.

To prove Theorem 2.1 we need some preliminary results.

LEMMA 2.3. Let $C$ be a union of $t \geq 3$ degenerate skew lines in $\mathbf{P}^{4}$ in a hyperplane $H$ and let $C^{\prime}$ be any degenerate curve in the liaison class of $C$. Then $C^{\prime}$ also lies in the hyperplane $H$.

Proof. Let $L$ be the linear form corresponding to $H$.

Claim 2.3.1. For all $n, \varphi_{n}(L): M(C)_{n} \rightarrow M(C)_{n+1}$ is the zero homomorphism (and similarly for $C^{\prime}$ ).

This is essentially the content of Remark 2.7 of [M1], but we include the proof since it is short.

We have the exact sequences

$$
0 \rightarrow \mathscr{I}_{C}(n) \rightarrow \mathcal{O}_{\mathbf{P}^{4}}(n) \rightarrow \mathcal{O}_{C}(n) \rightarrow 0
$$

and

$$
0 \rightarrow \mathcal{O}_{\mathbf{p}^{4}}(n) \rightarrow \mathscr{I}_{C}(n+1) \rightarrow \mathscr{I}_{C, H}(n+1) \rightarrow 0
$$

where the inclusion map in the second sequence is multiplication by $L$. Taking cohomology, these sequences yield

$$
\begin{aligned}
& \cdots \rightarrow M(C)_{n} \rightarrow 0 \rightarrow \cdots, \\
& \cdots \rightarrow 0 \rightarrow M(C)_{n+1} \rightarrow \cdots,
\end{aligned}
$$

and again the composition is $\varphi_{n}(L)$. The same proof works for $C^{\prime}$.

Claim 2.3.2. $\varphi_{0}\left(L^{\prime}\right): M(C)_{0} \rightarrow M(C)_{1}$ is never zero if $L^{\prime}$ is not a scalar multiple of $L$. 
For a general $L^{\prime}$ this follows from the long exact sequence associated to (1.1.5):

$$
\begin{aligned}
0 & \rightarrow H^{0}\left(\mathscr{I}_{C}\right) \rightarrow H^{0}\left(\mathscr{I}_{C}(1)\right) \rightarrow H^{0}\left(\mathscr{I}_{C \cap H^{\prime}}(1)\right) \\
& \rightarrow M(C)_{0} \stackrel{\varphi_{0}\left(L^{\prime}\right)}{\rightarrow} M(C)_{1} \rightarrow \cdots
\end{aligned}
$$

One checks from (1.1.4) that $\operatorname{dim} M(C)_{0}=t-1$, and clearly $h^{0}\left(\mathscr{I}_{C}\right)=0$ and $h^{0}\left(\mathscr{I}_{C}(1)\right)=1$. In order for $\varphi_{0}\left(L^{\prime}\right)$ to be zero, then, we would need $h^{0}\left(\mathscr{I}_{C \cap H^{\prime}}(1)\right)=t$. But $h^{0}\left(\mathscr{I}_{C \cap H^{\prime}}(1)\right)$ counts the (vector space) dimension of the family of hyperplanes (i.e. 2-planes) in $H^{\prime}=\mathbf{P}^{3}$ containing the points of $C \cap H^{\prime}$, which is at most 2 (which happens when the points of $C \cap H^{\prime}$ are collinear).

The only other possibility is that $L^{\prime}$ vanishes on one component of $C$, say $\lambda$. Then using the long exact sequences associated to (1.1.6) (taking $n=0$ ) we get

$$
\begin{aligned}
\cdots & \rightarrow H^{0}\left(\mathscr{I}_{Y}\right) \rightarrow H^{0}\left(\mathscr{O}_{\lambda}\right) \rightarrow M(C)_{0} \rightarrow M(Y)_{0} \rightarrow \cdots, \\
0 & \rightarrow H^{0}\left(\mathscr{I}_{Y}\right) \rightarrow H^{0}\left(\mathscr{I}_{C}(1)\right) \rightarrow H^{0}\left(\mathscr{I}_{C \cap H^{\prime}}(1)\right) \rightarrow M(Y)_{0} \\
& \rightarrow M(C)_{1} \rightarrow \cdots .
\end{aligned}
$$

Now, $h^{0}\left(\mathscr{I}_{Y}\right)=0$ and $h^{0}\left(\mathscr{I}_{C}(1)\right)=1$. There is exactly one plane in $H^{\prime}=\mathbf{P}^{3}$ containing $C \cap H^{\prime}$, namely the plane $H \cap H^{\prime}$. (Recall that $H^{\prime}$ contains a component of $C$.) Hence $h^{0}\left(\mathscr{I}_{C \cap H^{\prime}}(1)\right)=1$ and the map $M\left(Y_{0}\right) \rightarrow M(C)_{1}$ is an injection. Then composing, we get $\operatorname{ker} \varphi_{0}\left(L^{\prime}\right) \cong$ $H^{0}\left(\mathcal{O}_{\lambda}\right)$, which is 1 -dimensional. Since $\operatorname{dim} M(C)_{0}=t-1 \geq 2$, we are finished with Claim 2.3.2.

Now let $C^{\prime}$ be any degenerate curve, and say $L_{1}$ vanishes on $C^{\prime}$. If $C^{\prime}$ is evenly linked to $C$ we look at the first non-trivial component of $M\left(C^{\prime}\right)$, say in degree $m$. Let $\psi_{m}: S_{1} \rightarrow \operatorname{Hom}\left(M\left(C^{\prime}\right)_{m}, M\left(C^{\prime}\right)_{m+1}\right)$. From Claim 2.3.1 we have $\psi_{m}\left(L_{1}\right)$ is zero. From the ideas of $\S 1$ we know that $\psi_{m}\left(L_{1}\right)=\varphi_{0}\left(L_{1}\right)$ (abusing notation). But we have just seen that $\varphi_{0}\left(L^{\prime}\right)$ is zero if and only if $L^{\prime}$ is a scalar multiple of $L$. Therefore $L_{1}$ is a scalar multiple of $L$ and $C$ and $C^{\prime}$ are in the same hyperplane.

If $C^{\prime}$ is oddly linked to $C$ we look at the "last" map in $M\left(C^{\prime}\right)$ rather than $\psi_{m}$ as above, and we again compare it with $\varphi_{0}$. As before, this will be zero for scalar multiples of the linear form $L$ and non-zero otherwise. Since ranks are preserved under transposes, a similar argument works here and we are done Lemma 2.3.

LEMMA 2.4. Let $C$ be as above and let $C^{\prime}$ be a non-degenerate union of $s$ skew lines in $\mathbf{P}^{4}$. Then $C$ is not linked to $C^{\prime}$. 
Proof. Let $\psi_{0}: S_{1} \rightarrow \operatorname{Hom}\left(M\left(C^{\prime}\right)_{0}, M\left(C^{\prime}\right)_{1}\right)$. A very minor modification of the argument in Claim 2.3.2 above shows that $\psi_{0}\left(L^{\prime}\right)$ is never zero except in the case where $s=3$ and $L^{\prime}$ vanishes on the trisecant of $C^{\prime}$ (see $\S 3)$. Either way $M(C)$ cannot be isomorphic to a shift of $M\left(C^{\prime}\right)$ or $M\left(C^{\prime}\right)^{\vee}$ so the result follows immediately

Proof of Theorem 2.1. We have thus far shown that any set $C^{\prime}$ of skew lines in the liaison class of a degenerate set $C$ of skew lines must lie in the same hyperplane. It is conceivable, through, that to reach $C^{\prime}$ from $C$ one must "leave" the hyperplane. That is, they might be linked in $\mathbf{P}^{4}$ but not in $\mathbf{P}^{3}$. We now show that this does not happen.

Without loss of generality let the hyperplane be given by the vanishing of $X_{4}$. Since $\varphi_{n}\left(X_{4}\right)$ is identically zero, $M(C)$ can be viewed as a $k\left[X_{0}, \ldots, X_{3}\right]$-module, as can $M\left(C^{\prime}\right)$. Since $C \sim C^{\prime}$ we have (up to shifts) either $M(C) \cong M\left(C^{\prime}\right)$ or $M(C) \cong M\left(C^{\prime}\right)^{\vee}$ as $k\left[X_{0}, \ldots, X_{4}\right]$-modules, so the same is true as $k\left[X_{0}, \ldots, X_{3}\right]$-modules. But then by Rao's theorem (cf. [R1]) $C \sim C^{\prime}$ in $\mathbf{P}^{3}$.

It is trivially true that if $C \sim C^{\prime}$ in $\mathbf{P}^{3}$ then $C \sim C^{\prime}$ in $\mathbf{P}^{4}$. Therefore we are done.

REMARK 2.5. If $C$ consists of two skew lines in $\mathbf{P}^{4}$ then this argument fails since $M(C)=k$ (in degree 0 ) so there are no non-trivial maps to study. One may conjecture, though, that Theorem 2.1 remains true in this case: if $C^{\prime}$ is another pair of skew lines then $C \sim C^{\prime}$ if and only if they lie in the same hyperplane. In fact, it seems reasonable to conjecture that if $C$ is any degenerate, non-Cohen-Macaulay curve in $\mathbf{P}^{4}$ and $C^{\prime}$ is a degenerate curve in the liaison class of $C$ in $\mathbf{P}^{4}$ then they lie in the same hyperplane and are linked inside that hyperplane.

REMARK 2.6. The techniques which we have used here can clearly be used for degenerate disjoint unions of $t$ lines in any projective space $\mathbf{P}^{n}$, provided $M(C)_{1}$ is non-zero.

3. Three non-degenerate skew lines. For the remainder of this paper we assume that $C$ is a non-degenerate union of $t$ skew lines in $\mathbf{P}^{4}$. The case $t=3$ is the only one for which we cannot give a full answer using these techniques, although we will give a partial result.

Observe that for $t=3$ we have from (1.1.4) that

$$
\operatorname{dim} M(C)_{i}= \begin{cases}2, & i=0 \\ 1, & i=1 \\ 0, & i \neq 0,1\end{cases}
$$


(The last also uses the easily-verifiable fact that $C$ imposes $3(d+1)$ conditions on hypersurfaces of degree $d$ for $d \geq 2$ since $C$ is non-degenerate.) From these dimensions we expect that for the generic $L \in S_{1}$, rk $\varphi_{0}(L)=1$ (where again $\varphi_{0}: S_{1} \rightarrow \operatorname{Hom}\left(M(C)_{0}, M(C)_{1}\right)$ ) and that those $L$ for which this fails form an algebraic set of codimension 2 and degree 1 in $S_{1}$ (or in $\left.\mathbf{P} S_{1}=\left(\mathbf{P}^{4}\right)^{*}\right)$ (cf. [M1], Lemma 1.4).

The techniques described in $\$ 1$ show that this is indeed the case, and from this we can say something about liaison:

Proposition 3.1. Let $C$ be as above and let $C^{\prime}$ be any union of $s$ skew lines in $\mathbf{P}^{4}$. If $C \sim C^{\prime}$ then

(a) $C$ and $C^{\prime}$ are evenly linked,

(b) $s=3$,

(c) $C$ and $C^{\prime}$ share a common trisecant.

Proof. For any union $Z$ of $t$ skew lines we have $\operatorname{dim} M(Z)_{0}=t-1$, and we know that if $t=2$ then $M(Z) \cong k$. Therefore $M(C)^{\vee}$ does not correspond to any union of skew lines, and by Corollary 1.2 we are done with (a). Having this, (b) is trivial.

Now, observe that $C$ has a unique trisecant, say $\alpha$. We shall show that this is the dual of the plane in $\left(\mathbf{P}^{4}\right)^{*}$ which we anticipated above. If $L \in S_{1}$ does not vanish on any component of $C$ and if $H$ is the corresponding hyperplane then using (1.1.5) we have $\operatorname{rk} \varphi_{0}(L)=0$ if and only if $h^{0}\left(\mathscr{I}_{C \cap H}(1)\right)=2$, which holds if and only if $L$ vanishes on $\alpha$ (so $C \cap H$ consists of three collinear points). This continues to hold for those $L$ which vanish on $\alpha$ and a component of $C$ since the set of $L$ for which $\varphi_{0}(L)$ drops rank is closed.

If $L \in S_{1}$ vanishes on a component of $C$ but not on $\alpha$ then using (1.1.6) one checks that $\operatorname{rk} \varphi_{0}(L)=1$. Therefore, $\alpha$ is an invariant of the even liaison class of $C$, and we are done.

It is very unlikely that the converse of Proposition 3.1 is true.

4. Four general skew lines. From now on we will consider only a general collection $C$ of $t$ skew lines (i.e. an open subset of the appropriate product of Grassmannians $G(2,5)^{t}$ where $G(2,5)$ is the Grassmannian of lines in $\mathbf{P}^{4}$ ). For $t=4$ this will mean that $C$ has no quadrisecants and finitely many trisecants, all of which are disjoint. Let $C=\bigcup_{i=1}^{4} \lambda_{i}$ be such a curve. We adopt the notation $\left[\lambda_{i}, \lambda_{j}\right]$ to denote the linear space spanned by $\lambda_{i}$ and $\lambda_{j}$. 
LEMMA 4.1. (a) Any three components of $C$ are non-degenerate.

(b) There are exactly 4 trisecants, any 3 of which are non-degenerate.

(c) The union of the trisecants is directly linked to $C$.

(d) $h^{0}\left(\mathscr{I}_{C}(2)\right)=3$.

Proof. Three skew lines in $\mathbf{P}^{4}$ have either a unique trisecant (when they are non-degenerate) or infinitely many (when they are degenerate). By hypothesis, then, (a) and the first part of (b) are done. Because the trisecants are assumed disjoint, one sees that the union of $C$ with the trisecants is a so-called double-four configuration, which is a complete intersection. (From (1.1.4) one checks that $C$ lies on at least 3 independent quadrics, which can be chosen to meet properly, and all 3 contain the trisecants by Bezout's theorem. But the complete intersection of these quadrics has degree 8 and so must be the union of $C$ and the trisecants. See [DR] for more on double-fours.) Therefore we are done with (c) and (d) also. As for the second part of (b), note that the hyperplane spanned by two trisecants coincides with one of the hyperplanes $\left[\lambda_{i}, \lambda_{j}\right]$, so the proof is finished.

Now, let $C^{\prime}$ be the union of the trisecants. The goal of this section is to prove the following:

THEOREM 4.2. $C^{\prime}$ is the only other union of skew lines in the liaison class of $C$.

Proof. Observe first that $\operatorname{dim} M(C)_{0}=\operatorname{dim} M(C)_{1}=3$ and all other components are zero. (Recall that $h^{0}\left(\mathscr{I}_{C}(2)\right)=3$ from Lemma 4.1 and use the exact sequence (1.1.4).) Hence any set of skew lines which can hope to be evenly or oddly linked to $C$ must have exactly four components.

Now, we shall show how to recover the double-four $C \cup C^{\prime}$ from $M(C)$, proving that $C$ and $C^{\prime}$ are unique. To begin, note that we expect the set of $L$ 's for which $\operatorname{rk} \varphi_{0}(L)<3$ to form a cubic hypersurface in $\left(\mathbf{P}^{4}\right)^{*}$; and since one can easily construct a hyperplane which meets $C$ in four non-coplanar points (so $h^{0}\left(\mathscr{I}_{C \cap H}(1)\right)=0$ and $\operatorname{rk} \varphi_{0}(L)=3$ by (1.1.5)) this must in fact be correct. (cf. [M1], Lemma 1.4). Call this three-fold $T$.

We are interested in knowing something about the subvarieties of $T$. An amusing fact (which we will not need) is that $T$ is ruled, in the sense that for any point $P$ of $T$ there exists (at least) one line contained in $T$ which passes through $P$. The main part of the argument is the following: let $H$ be the hyperplane corresponding to $P \in T \subset\left(\mathbf{P}^{4}\right)^{*}$. If $H$ contains 
a component $\lambda_{i}$ of $C$ then there is actually a 2-plane in $T$ containing $P$ (see below). If $H$ meets $C$ properly, then the fact that $P \in T$ means that $h^{0}\left(\mathscr{I}_{C \cap H}(1)\right)>0$, so the four points of $C \cap H$ lie in some 2-plane $\Lambda$. But there is a pencil of hyperplanes containing $L$, corresponding to a line on $T$ which contains $P$.

It is more important in the present context to study the 2-planes in $T$. Any such 2-plane is dual to a line in $\mathbf{P}^{4}$. What may these lines be? A partial result is the following:

Claim 4.2.1. (a) The 2-plane dual to a component $\lambda_{i}$ of $C$ lies in $T$.

(b) The 2-plane dual to a trisecant of $C$ lies in $T$.

Part (a) follows from the exact sequences (1.1.6), taking $n=0$, and one observation: let $L$ be a linear form vanishing on one component, say $\lambda$, and let $H$ be the corresponding hyperplane. Since $h^{0}\left(\mathcal{O}_{\lambda}\right)=1$, the exact sequences (1.1.6) will give us $\operatorname{dim} \operatorname{ker}\left(\varphi_{0}(L)\right)=1$ (and so rk $\varphi_{0}(L)$ =2) once we show that $h^{0}\left(\mathscr{I}_{C \cap H}(1)\right)=0$. But $C \cap H$ consists of the line $\lambda$ and three points. If $h^{0}\left(\mathscr{I}_{C \cap H}(1)\right)>0$ then there is a 2-plane containing $\lambda$ and these three points. This 2-plane then contains the three trisecants of $C$ which involve $\lambda$, so these trisecants are not disjoint. This contradicts the original hypothesis on $C$.

Similarly, part (b) follows from the exact sequence (1.1.5), again with $n=0$. (Here we use the fact that given any three collinear points and one other point, they are coplanar.) This completes the proof of Claim 4.2.1.

Now, consider the set of linear forms $L$ for which $\operatorname{rk} \varphi_{0}(L)=1$. We shall show that the projectivization of this set is a union of six points.

Let $Y=\lambda_{1} \cup \lambda_{2}$ and $Z=\lambda_{3} \cup \lambda_{4}$ and let $L$ be a linear form vanishing on $Y$. Consider the following two exact sequences (in analogy with (1.1.6)):

$$
\begin{gathered}
0 \rightarrow \mathscr{I}_{C} \rightarrow \mathscr{I}_{Z} \rightarrow \mathcal{O}_{Y} \rightarrow 0, \\
0 \rightarrow \mathscr{I}_{Z} \rightarrow \mathscr{I}_{C}(1) \rightarrow \mathscr{I}_{C \cap H}(1) \rightarrow 0,
\end{gathered}
$$

where again $H$ is the hyperplane corresponding to $L$. Since $h^{0}\left(\mathcal{O}_{Y}\right)=2$ and all the other global sections are zero, the composition gives $\operatorname{dim} \operatorname{ker}\left(\varphi_{0}(L)\right)=2$, so $\operatorname{rk} \varphi_{0}(L)=1$. The same can be done for $Y$ equal to any pair of components of $C$, so projectivizing we get the six points of $T$ dual to the six hyperplanes $\left[\lambda_{i}, \lambda_{j}\right]$.

We now claim that these are the only points where $\operatorname{rk} \varphi_{0}(L)=1$. If $L$ vanishes on exactly one $\lambda_{i}$ then we have already seen that $\operatorname{rk} \varphi_{0}(L)=2$. If $L$ does not vanish on any $\lambda_{i}$ then from (1.1.5) we get $\operatorname{rk} \varphi_{0}(L) \geq 2$ since $h^{0}\left(\mathscr{I}_{C \cap H}(1)\right) \leq 1$. (This latter is true since $C$ has no quadrisecant.) 
It follows that from $M(C)$ we can recover the six hyperplanes $\left[\lambda_{i}, \lambda_{j}\right]$. Intersecting three of these at a time gives a line in $\mathbf{P}^{4}$, so we get $20=\operatorname{comb}(6,3)$ distinguished lines. Four are the original components of $C$ (e.g. $\left.\lambda_{1}=\left[\lambda_{1}, \lambda_{2}\right] \cap\left[\lambda_{1}, \lambda_{3}\right] \cap\left[\lambda_{1}, \lambda_{4}\right]\right)$ and four are the trisecants (e.g. the trisecant to $\lambda_{1}, \lambda_{2}$ and $\lambda_{3}$ is $\left.\left[\lambda_{1}, \lambda_{2}\right] \cap\left[\lambda_{1}, \lambda_{3}\right] \cap\left[\lambda_{2}, \lambda_{3}\right]\right)$.

Unfortunately, there are 12 other lines which we must "weed out" in order to recover the double-four. Each of these 12 lines passes through two vertices of the double-four. For example, we will check that the diagonal line in Figure 1 is the intersection $\left[\lambda_{1}, \lambda_{2}\right] \cap\left[\lambda_{2}, \lambda_{3}\right] \cap\left[\lambda_{1}, \lambda_{4}\right]$.

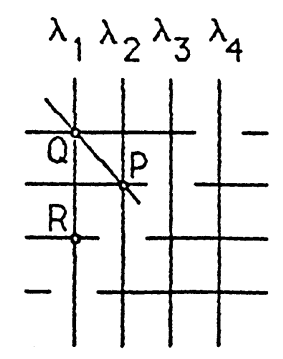

FigURE 1

Note first that $\left[\lambda_{1}, \lambda_{2}\right] \cap\left[\lambda_{2}, \lambda_{3}\right]$ is the plane spanned by $\lambda_{2}$ and the trisecant to $\lambda_{1}, \lambda_{2}$ and $\lambda_{3}$. Intersecting this with $\left[\lambda_{1}, \lambda_{4}\right]$ gives a line. (Otherwise it is the same plane as $\left[\lambda_{1}, \lambda_{2}\right] \cap\left[\lambda_{1}, \lambda_{4}\right]$, which contains $\lambda_{1}$, which would mean that $\lambda_{1}$ and $\lambda_{2}$ are coplanar and hence not disjoint.) But one can check that $\lambda_{2}$ meets $\left[\lambda_{1}, \lambda_{4}\right]$ at $P$ (since any three $\lambda_{i}$ are non-degenerate) and the trisecant to $\lambda_{1}, \lambda_{2}$ and $\lambda_{3}$ meets $\left[\lambda_{1}, \lambda_{4}\right]$ at $Q$ (since if this trisecant lay in $\left[\lambda_{1}, \lambda_{4}\right]$ there would be three trisecants in this hyperplane, contadicting Lemma 4.1). Therefore, the intersection $\left[\lambda_{1}, \lambda_{2}\right]$ $\cap\left[\lambda_{2}, \lambda_{3}\right] \cap\left[\lambda_{1}, \lambda_{4}\right]$ is the diagonal line $[P, Q]$ in Figure 1 .

Using this type of reasoning, one can find the 12 lines and describe them as follows. Let $P$ and $Q$ be any vertices in Figure 1 which are not on the same line but which are opposite vertices of a "quadrilateral". There are 6 such quadrilaterals and hence 12 such choices of $P$ and $Q$ (ordering is not important here). Then $[P, Q]$ is one of the 12 lines.

Now we can finally recover the double-four from $M(C)$. We have seen (Claim 4.2.1) that the 2-planes dual to the $\lambda_{i}$ and to the trisecants lie on $T$. We shall show that the duals to the other 12 lines do not lie on $T$, which distinguishes the double-four among the 20 lines and concludes the proof. To do this, for any of the other 12 lines we show that there exists a hyperplane containing it which meets $C$ in exactly four points which are non-coplanar (and then invoke (1.1.5)). 
For convenience we shall examine the line $[P, Q]$ of Figure 1 . We first claim that this line does not lie in $\left[\lambda_{3}, \lambda_{4}\right]$. For if it did, then $Q$ would lie in $\left[\lambda_{3}, \lambda_{4}\right]$. But the point $R$ also lies in $\left[\lambda_{3}, \lambda_{4}\right]$ since it is on the trisecant to $\lambda_{1}, \lambda_{3}$ and $\lambda_{4}$, so $[Q, R]=\lambda_{1}$ must lie in $\left[\lambda_{3}, \lambda_{4}\right]$. This contradicts Lemma 4.1 (a) that no three of the $\lambda_{i}$ are the same hyperplane.

Hence the line $[P, Q]$ meets $\left[\lambda_{3}, \lambda_{4}\right]$ in a point, say $P_{1}$. Let $H$ be a hyperplane containing $[P, Q]$ and meeting $C$ properly. Then $H$ meets $\lambda_{3}$ and $\lambda_{4}$ in points $P_{3}$ and $P_{4}$, respectively. Since $[P, Q], P_{3}$ and $P_{4}$ determine $H$, clearly we may choose $H$ so that $P_{1}, P_{3}$ and $P_{4}$ are non-collinear. But then for such an $H$ the lines $[P, Q]$ and $\left[P_{3}, P_{4}\right]$ are skew so $C \cap H=P \cup Q \cup P_{3} \cup P_{4}$ are four non-coplanar points.

5. Five or more general lines. We now let $C=\bigcup_{i=1}^{t} \lambda_{i}$ be a general collection of $t \geq 5$ skew lines in $\mathbf{P}^{4}$. In this case, one generality assumption that we make is that the curve $C$ have maximal rank, i.e. that the restriction map $\rho_{n}: H^{0}\left(\mathcal{O}_{\mathbf{P}^{4}}(n)\right) \rightarrow H^{0}\left(\mathcal{O}_{C}(n)\right)$ be either injective or surjective for all $n$. (Equivalently, for all $n$ either $h^{0}\left(\mathscr{I}_{C}(n)\right)=0$ or $\operatorname{dim} M(C)_{n}$ $=0$.) This is valid since those sets of $t$ skew lines with maximal rank correspond to a dense Zariski open subset of $G(2,5)^{t}$ (cf. [HH]). Another assumption is that any three components of $C$ are non-degenerate.

Before stating the main theorem of this section, we recall a fact whose proof is a standard application of the geometry on a Grassmannian. I am grateful to Tony Horowitz for pointing this out to me and for several related conversations which proved useful for part of this section.

Lemma 5.1. Given six sufficiently general lines in $\mathbf{P}^{4}$, there are exactly five 2-planes meeting each line.

In this section we will prove the following (compare with Theorem $2.2(\mathrm{c}))$.

THEOREM 5.2. Let $C=\bigcup_{i=1}^{t} \lambda_{i}$ be a general collection of $t$ skew lines in $\mathbf{P}^{4}$, where $t \geq 5$. Then $C$ is not linked to any other disjoint union of lines in $\mathbf{P}^{4}$.

Proof. As usual, the first step is to compute the dimension of the components of $M(C)$. Because of maximal rank, we have $\operatorname{dim} M(C)_{n}= \begin{cases}t(n+1)-\operatorname{comb}(n+4,4), & t(n+1) \geq \operatorname{comb}(n+4,4), \\ 0, & t(n+1) \leq \operatorname{comb}(n+4,4) .\end{cases}$ 
We first consider the case of odd linkage. This means that we must ask whether $M(C)^{\vee}$ (with a suitable shift) can be the Hartshorne-Rao module of any union of skew lines. To do this, we recall a trick introduced in [M1].

For any curve $Y$, let $a_{n}=\operatorname{dim} M(Y)_{n}$ as long as either $\operatorname{dim} M(Y)_{n} \neq$ 0 or $h^{0}\left(\mathscr{I}_{Y}(n)\right)=0$, and $a_{n}$ is undefined otherwise. (If $Y$ has maximal rank then $h^{0}\left(\mathscr{I}_{Y}(n)\right)=0$ is enough.) Let $b_{n}=2 a_{n+1}-a_{n}-a_{n+2}$ as long as this is defined. In the case of skew lines of maximal rank, the last condition on $a_{n}$ allows one more $b_{n}$ in case $t(n+1)=\operatorname{comb}(n+4,4)$. Then one checks that for $C$ a collection of $t$ skew lines of maximal rank, $b_{n}=\operatorname{comb}(n+4,2)$ as long as it is defined. In particular, since $t \geq 5$ we have $b_{0}=6$, and if $t \geq 9$ we also have $b_{1}=10$.

Suppose $C$ were oddly linked to a disjoint union of lines $C^{\prime}$. We have similarly defined integers $b_{n}^{\prime}$ for $C^{\prime}$, and we get that the sequence $\left\{b_{n}^{\prime}\right\}$ is the reverse of the sequence $\left\{b_{n}\right\}$ (using Corollary 1.2). In particular, $b_{0}^{\prime}$ is the last $b_{n}$.

Of course $C^{\prime}$ need not have maximal rank, so there are more possibilities for the $b_{n}^{\prime}$. We have in particular

$$
b_{0}^{\prime}= \begin{cases}6, & h^{0}\left(\mathscr{I}_{C^{\prime}}(2)\right)=0, \\ 5, & h^{0}\left(\mathscr{I}_{C^{\prime}}(2)\right)=1, \\ 4, & h^{0}\left(\mathscr{I}_{C^{\prime}}(2)\right)=2, \\ 3, & h^{0}\left(\mathscr{I}_{C^{\prime}}(2)\right)=3 .\end{cases}
$$

Since $b_{0}^{\prime}$ is the last $b_{n}$ and $\left\{b_{n}\right\}$ is a strictly increasing sequence beginning with 6 , we immediately conclude that $b_{0}^{\prime}=6=b_{0}$ and $b_{0}$ is the only term in the sequence. Hence $h^{0}\left(\mathscr{I}_{C^{\prime}}(2)\right)=0$ and $t \leq 8$. Furthermore, using these facts and the explicit dimensions of $M(C)_{n}$ for $5 \leq t \leq 8$ we also see that no shift of $M(C)^{\vee}$ can be the Hartshorne-Rao module of a union of skew lines $C^{\prime}$ if $t=5,6$ or 8 .

We thus have only to show that a general set of 7 skew lines is not oddly linked to any set of skew lines. If $t=7$ we get $\operatorname{dim} M(C)_{0}=6$, $\operatorname{dim} M(C)_{1}=9, \operatorname{dim} M(C)_{2}=6$ and $\operatorname{dim} M(C)_{n}=0$ otherwise. Hence $C$ could in principle be linked to another union $C^{\prime}$ of 7 skew lines. We now rule this out. We will show that $\varphi_{1}: S_{1} \rightarrow \operatorname{Hom}\left(M(C)_{1}, M(C)_{2}\right)$ is incompatible with the $\varphi_{0}^{\prime}$ for any such $C^{\prime}$.

As usual, $\varphi_{0}^{\prime}$ would have to drop rank on the planes dual to the components of $C^{\prime}$. Since $\varphi_{0}^{\prime}$ is the transpose of $\varphi_{1}$, we now check that $\varphi_{1}$ does not drop rank on any planes in $\left(\mathbf{P}^{4}\right)^{*}$ and this will complete the question of odd liaison. The main step is the following claim. 
Claim. 5.2.1. Let $C=\lambda_{1} \cup \cdots \cup \lambda_{6} \cup \lambda$ and $Y=\lambda_{1} \cup \cdots \cup \lambda_{6}$. Let $L$ be a general linear form vanishing on $\lambda$ and $H$ the corresponding hyperplane. Then $\varphi_{1}(L): M(C)_{1} \rightarrow M(C)_{2}$ is surjective.

We have exact sequences (1.1.6):

$$
\begin{aligned}
0 & \rightarrow H^{0}\left(\mathscr{I}_{C}(1)\right) \rightarrow H^{0}\left(\mathscr{I}_{Y}(1)\right) \rightarrow H^{0}\left(\mathscr{O}_{\lambda}(1)\right) \rightarrow M(C)_{1} \\
& \rightarrow M(Y)_{1} \rightarrow \cdots, \\
0 & \rightarrow H^{0}\left(\mathscr{I}_{Y}(1)\right) \rightarrow H^{0}\left(\mathscr{I}_{C}(2)\right) \rightarrow H^{0}\left(\mathscr{I}_{C \cap H}(2)\right) \\
& \rightarrow M(Y)_{1} \rightarrow M(C)_{2} \rightarrow \cdots .
\end{aligned}
$$

One easily checks $h^{0}\left(\mathcal{O}_{\lambda}(1)\right)=2, \operatorname{dim} M(C)_{1}=9, \operatorname{dim} M(Y)_{1}=7$, $\operatorname{dim} M(C)_{2}=6$ and $h^{0}\left(\mathscr{I}_{C}(2)\right)=0$. Less trivial is $h^{0}\left(\mathscr{I}_{C \cap H}(2)\right)=1$. To see this, note that $C \cap H$ consists of $\lambda$ together with the 6 points $H \cap \lambda_{i}$. But by Lemma 5.1, for general $L$ vanishing on $\lambda$ these 6 points are non-coplanar, and no four are collinear since a general $C$ has no quadrisecants. Hence these 6 points together with $\lambda$ impose 9 conditions on quadrics in $H$ and $h^{0}\left(\mathscr{I}_{C \cap H}(2)\right)=10-9=1$ as desired.

Now, $\varphi_{1}(L)$ is given by the composition $M(C)_{1} \rightarrow M(Y)_{1} \rightarrow M(C)_{2}$. The dimensions above imply that both maps are surjective, so $\varphi_{1}(L)$ is also surjective and we have proved Claim 5.2.1.

This says that if we want to find those $L$ for which $\varphi_{1}(L)$ drops rank, we need not consider those $L$ vanishing on just one component of $C$. If $\varphi_{1}$ is to drop rank on a 2-plane in $\left(\mathbf{P}^{4}\right)^{*}$, then the general such $L$ must meet $C$ transversely so the exact sequence (1.1.5) applies:

$$
\begin{aligned}
0 & \rightarrow H^{0}\left(\mathscr{I}_{C}(1)\right) \rightarrow H^{0}\left(\mathscr{I}_{C}(2)\right) \rightarrow H^{0}\left(\mathscr{I}_{C \cap H}(2)\right) \rightarrow M(C)_{1} \\
& \rightarrow M(C)_{2} \rightarrow \cdots .
\end{aligned}
$$

$C \cap H$ then consists of 7 points in the hyperplane $H$, and for $\varphi_{1}(L)$ to have less than maximal rank we need that these 7 points fail to impose independent conditions on quadrics. This can happen if and only if either

(1) all 7 points are coplanar,

(2) 6 of the points lie on a conic or

(3) 4 of the points are collinear.

We have already seen that no 4 can be collinear for a general $C$. The other two possibilities are ruled out by Lemma 5.1, since only finitely many planes can meet 6 general lines (corresponding to only a union of lines in $\left(\mathbf{P}^{4}\right)^{*}$ rather than a union of 2-planes) and no planes meet 7 general lines.

To summarize, we have shown that $\varphi_{1}$ cannot drop rank on any 2-plane in $\left(\mathbf{P}^{4}\right)^{*}$. Therefore $M(C)^{\vee}(-2)$ is not the Hartshorne-Rao module of any union of skew lines, and $C$ is not oddly linked to any other union of skew lines. 
We now turn to even liaison. Our goal is to show that for $t \geq 5 \mathrm{C}$ can be recovered from $M(C)$. For $t \geq 6$ the argument is much the same as above: the linear forms $L$ vanishing on a component of $C$ drop rank, so we get correspondingly a union of $t 2$-planes in $\left(\mathbf{P}^{4}\right)^{*}$. Since no other 2-plane in $\left(\mathbf{P}^{4}\right)^{*}$ corresponds to rank-dropping $L$ (by Lemma 5.1 and the usual exact sequence) we recover $C$ from this union of 2-planes.

We thus have only to consider the case $t=5$. For this we make one more generality assumption: if $\lambda$ is any trisecant (say meeting $\lambda_{1}, \lambda_{2}$ and $\lambda_{3}$ ) then $\lambda$ does not lie in the hyperplane spanned by the other two lines (in this case $\lambda_{4}$ and $\lambda_{5}$ ). To justify this we start with a general collection of four lines as described in $\S 4$ and check that to fail to have the desired property is a (union of) closed condition(s) on the Grassmannian of lines $G(2,5)$. For example, let us check in Figure 2 that the set of lines $\lambda_{5}$ for which the trisecant to $\lambda_{3}, \lambda_{4}$ and $\lambda_{5}$ lies in the hyperplane $\left[\lambda_{1}, \lambda_{2}\right]$ is contained in a closed set in $G(2,5)$.

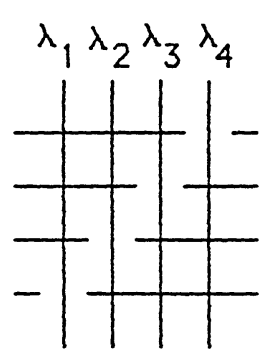

FIGURE 2

In order to be such a trisecant, in particular it must lie in the hyperplane $\left[\lambda_{3}, \lambda_{4}\right]$. If it also lies in $\left[\lambda_{1}, \lambda_{2}\right]$ then it lies in the plane of intersection of these two hyperplanes. Since any 3 of the 5 lines are non-degenerate, this plane meets $\lambda_{3}$ and $\lambda_{4}$ each in a point, thus uniquely determining the line which must be the trisecant. So $\lambda_{5}$ must meet this line, which is a closed condition.

Now, let us consider again the linear forms $L$ for which $\varphi_{0}(L)$ drops rank. As before, those linear forms vanishing on a component of $C$ drop rank, giving a union of five 2-planes in $\left(\mathbf{P}^{4}\right)^{*}$. We now claim that no other 2-planes in $\left(\mathbf{P}^{4}\right)^{*}$ will correspond to rank-dropping $L$, so as before $C$ can be recovered from $M(C)$ and we will be done. To prove this, it is enough to prove that there is no line $\lambda$ in $\mathbf{P}^{4}$ with the property that every hyperplane through $\lambda$ meeting $C$ properly meets $C$ in 5 coplanar points. Call this property (A). 
Note first that given five general lines, the set of 2-planes in $\mathbf{P}^{4}$ meeting all five is a curve in $G(3,5)$. We shall show that this is incompatible with the existence of a line with property (A).

The main claim is that no matter how $\lambda$ is chosen, there will exist two components $\lambda_{i}$ and $\lambda_{j}$ of $C$ such that $\lambda$ does not meet either $\lambda_{i}$ or $\lambda_{j}$ and does not lie in the hyperplane $\left[\lambda_{i}, \lambda_{j}\right.$ ]. If $\lambda$ is a trisecant then this follows from the above generality assumption and the fact that a general choice of five lines has no quadrisecant. Otherwise, there will be at least three components $\lambda_{i}, \lambda_{j}$ and $\lambda_{k}$ which do not meet $\lambda$. But the intersection of the three hyperplanes $\left[\lambda_{i}, \lambda_{j}\right],\left[\lambda_{j}, \lambda_{k}\right]$ and $\left[\lambda_{i}, \lambda_{k}\right]$ is the trisecant to $\lambda_{i}$, $\lambda_{j}$ and $\lambda_{k}$, so at least one of these three hyperplanes must fail to contain $\lambda$.

Assume then that $\lambda$ does not meet either of $\lambda_{i}$ and $\lambda_{j}$ and that $\lambda$ does not lie in the hyperplane $\left[\lambda_{i}, \lambda_{j}\right]$. Suppose further that $\lambda$ has property (A).

Choosing general points $P_{i} \in \lambda_{i}$ and $P_{j} \in \lambda_{j}$, together with $\lambda$, uniquely determines a hyperplane. If we can show that choosing general points $P_{i} \in \lambda_{i}$ and $P_{j} \in \lambda_{j}$, together with $\lambda$, uniquely determines a hyperplane which meets $C$ in exactly five points then we are done: by property (A) there is a 2-plane through these five points (two of which are $P_{i}$ and $P_{j}$ ) and varying the points $P_{i}$ and $P_{j}$ gives a 2-dimensional family of such 2-planes in $G(3,5)$ rather than a curve.

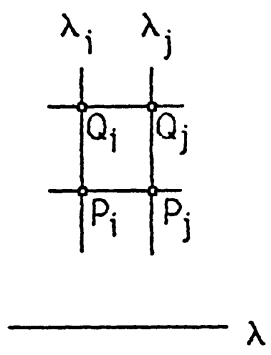

\section{FIGURE 3}

Let $Q_{i} \in \lambda_{i}$ and $Q_{j} \in \lambda_{j}$ be general points (see Figure 3 ). If the hyperplane $\left[\lambda, P_{i}, P_{j}\right]$ is the same as the hyperplane $\left[\lambda, Q_{i}, Q_{j}\right]$ then this hyperplane contains $\lambda, \lambda_{i}$ and $\lambda_{j}$, contradicting our assumption, so these hyperplanes must be different. But the general hyperplane through $\lambda$ can be determined in this way (since $\lambda$ does not meet either $\lambda_{i}$ or $\lambda_{j}$ ) and the general hyperplane through $\lambda$ does not contain any component of $C$. Hence we have accomplished our goal of the previous paragraph and hence have proved Theorem 5.2. 


\section{REFERENCES}

[B] G. Bolondi, On the classification of curves linked to two skew lines, preprint.

[BM] G. Bolondi and J. Migliore, Classification of maximal rank curves in the liaison class $\mathbf{L}_{n}$, in preparation.

[C] N. Chiarli, Completeness and Non-Speciality of Linear Series on Space Curves and Deficiency of the Corresponding Linear Series on Normalization, in The Curves Seminar at Queen's, vol. III, Queen's Papers in Pure and Applied Mathematics (1984).

[DR] B. Dayton and L. Roberts, The Seminormalization of an Abstract Configuration of Lines, Northeastern Ill. Univ. Technical Report No. 1984-11-1.

[H] C. Huneke, Numerical invariants of liaison classes, Invent. Math., 75 (1984), 301-325.

[HH] R. Hartshorne and A. Hirschowitz, Droites en position générale dans l'espace projectif, in Algebraic Geometry Proceedings (La Rábida, 1981), SLN 961 (1982).

[KMU]A. Kustin, M. Miller and B. Ulrich, Linkage theory for algebras with pure resolutions, to appear in J. Algebra.

[LR] R. Lazarsfeld and P. Rao, Linkage of General Curves of Large Degree, in Algebraic Geometry-Open Problems (Ravello, 1982), SLN 997 (1983).

[M1] J. Migliore, Geometric invariants for liaison of space curves, to appear in J. Algebra, 99 (1986).

[M2] - On linking double lines, Trans. Amer. Math. Soc., 294 (1986), 177-185.

[PS] C. Peskine and L. Szpiro, Liaison des variétés algébriques, Invent. Math., 26 (1974), 271-302.

[R1] P. Rao, Liaison among curves in $\mathbf{P}^{3}$, Invent. Math., 50 (1979), 205-217.

[R2] , Liaison equivalence classes, Math. Ann., 258 (1981), 169-173.

[S1] P. Schenzel, Notes on liaison and duality, J. Math. of Kyoto Univ., 22 (1982), 485-498.

[Su] P. Schwartau, Liaison Addition and Monomial Ideals, Ph.D. thesis, Brandeis University (1982).

[U] B. Ulrich, Rings of invariants and linkage of determinantal ideals, to appear in Math. Ann.

Received May 9, 1986.

DREW UNIVERSITY

MADISON, NJ 07940 


\section{PACIFIC JOURNAL OF MATHEMATICS EDITORS}

V. S. VARADARAJAN (Managing Editor) University of California Los Angeles, CA 90024 HERBERT ClEMENS University of Utah Salt Lake City, UT 84112

R. FINN

Stanford University

Stanford, CA 94305

\section{HERMANN FLASCHKA} University of Arizona Tucson, AZ 85721

RAMEsh A. GANGolli University of Washington Seattle, WA 98195

VAUghan F. R. JONES

University of California Berkeley, CA 94720

\section{ROBION KIRBY}

University of California

Berkeley, CA 94720

C. C. MOORE

University of California

Berkeley, CA 94720

HAROLD STARK

University of California, San Diego

La Jolla, CA 92093

\section{ASSOCIATE EDITORS}
R. ARENS
E. F. BECKENBACH
B. H. NEUMANN
F. WOLF
K. YOSHIDA (1906-1982)

\section{SUPPORTING INSTITUTIONS}

UNIVERSITY OF ARIZONA

UNIVERSITY OF BRITISH COLUMBIA

CALIFORNIA INSTITUTE OF TECHNOLOGY

UNIVERSITY OF CALIFORNIA

MONTANA STATE UNIVERSITY

UNIVERSITY OF NEVADA, RENO

NEW MEXICO STATE UNIVERSITY

OREGON STATE UNIVERSITY
UNIVERSITY OF OREGON

UNIVERSITY OF SOUTHERN CALIFORNIA

STANFORD UNIVERSITY

UNIVERSITY OF HAWAII

UNIVERSITY OF TOKYO

UNIVERSITY OF UTAH

WASHINGTON STATE UNIVERSITY

UNIVERSITY OF WASHINGTON 


\section{Pacific Journal of Mathematics}

Vol. 130, No. $1 \quad$ September, 1987

K. Adachi, Continuation of bounded holomorphic functions from certain subvarieties to weakly pseudoconvex domains $\ldots \ldots \ldots \ldots \ldots \ldots \ldots$

Erazm Jerzy Behr, Enveloping algebras of Lie superalgebras ........... 9

Dong M. Chung, Scale-invariant measurability in abstract Wiener spaces . . . 27

Peter Gerard Dodds and Bernardus de Pagter, Algebras of unbounded

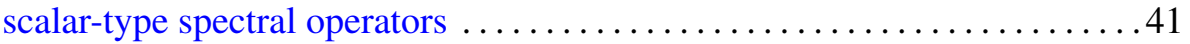

Wu-Yi Hsiang and Hsueh-Ling Huynh, Generalized rotational

hypersurfaces of constant mean curvature in the Euclidean spaces. II . . . .75

Harvey Bayard Keynes and M. Sears, Time changes for $\mathbf{R}^{n}$ flows and suspensions ..................................... 97

Frances Kirwan, Ronnie Lee and Steven Howard Weintraub, Quotients

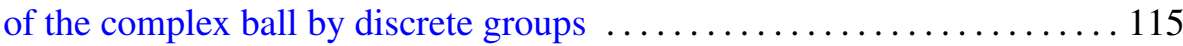

Magnhild Lien, Groups of knots in homology 3-spheres that are not

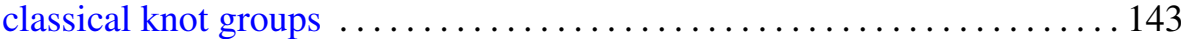

Juan Carlos Migliore, Liaison of a union of skew lines in $\mathbf{P}^{4} \ldots \ldots \ldots \ldots 153$

Jesper M. Møller, Spaces of sections of Eilenberg-Mac Lane fibrations . . . . 171 Daniel Ullman, A generalization of a theorem of Atkinson to noninvariant

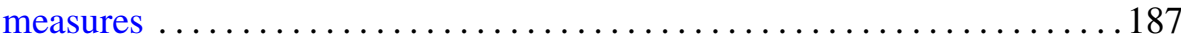

Kohhei Yamaguchi, Operations which detect $\mathscr{P}^{1}$ in odd primary connective

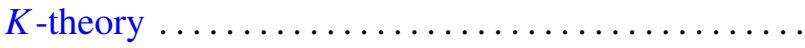

